\title{
$G$-Band Period-Luminosity Relation For Galactic Cepheids Based on Gaia DR1 Measurements
}

\author{
Chow-Choong Ngeow ${ }^{1}$, Anupam Bhardwaj ${ }^{2,3}$ and Shashi M. Kanbur ${ }^{4}$ \\ ${ }^{1}$ Graduate Institute of Astronomy, National Central University, Jhongli 32001, Taiwan \\ email: cngeow@astro.ncu.edu.tw \\ ${ }^{2}$ Department of Physics \& Astrophysics, University of Delhi, Delhi 110007, India \\ ${ }^{3}$ European Southern Observatory, Karl-Schwarzschild-Straße 2, Garching 85748, Germany \\ ${ }^{4}$ Department of Physics, SUNY Oswego, Oswego, NY 13126, USA
}

\begin{abstract}
Classical Cepheids (hereafter Cepheids) are important standard candle as they obey the famous period-luminosity (PL) relation. Parallax measurements from Gaia offer a unique opportunity to derive or calibrate the PL relations for Galactic Cepheids, as traditionally their distances were measured via different methods. In this work, we attempted to derive the Gaia $G$-band PL relation based on the Gaia Data Release 1 (DR1) measurements. We adopted the inferred distances provided by Astraatmadja \& Bailer-Jones (2016), calculated using two priors in a Bayesian analysis, and cross-matched to known Galactic Cepheids. The resulting $G$-band PL relation, however, exhibits a much larger scatter than expected. Hence the inferred distances based on the Gaia DR1 parallaxes are not suitable for calibrating the Galactic PL relation, and future Data Releases with improved parallax measurements are desirable.
\end{abstract}

Keywords. Cepheids, distance scale, stars: distances

\section{Introduction and Motivation}

Period-luminosity (PL) relation for classical Cepheids is an important rung on the extra-galactic distance ladder for the measurement of distances to nearby galaxies and hence the determination of the Hubble constant. Therefore it is desirable to calibrate the PL relation using Gaia's parallaxes (Casertano et al. 2016). The Gaia's Data Released 1 (DR1) includes the Gaia's G-band mean magnitudes and parallaxes $(p)$ based on the Tycho-Gaia Astrometric Solution (TGAS, hereafter DR1 parallax) for $\sim 2$ million stars brighter than $\sim 12$ mag. Lindegren et al. (2016) and Casertano et al. (2016) compare the DR1 parallaxes to parallaxes calculated from an adopted PL relation for $\sim 141$ and $\sim 212$ Galactic Cepheids, respectively. They found good global agreements between the two sets of parallaxes. Hence, the goal of this work is attempted to derive the Gaia's $G$ band PL relation for Galactic Cepheids based on the Gaia DR1 data, in order to evaluate the performance of DR1 parallaxes in deriving the Cepheid PL relations.

\section{Data, Method and Results}

Astraatmadja \& Bailer-Jones (2016a, hereafter ABJ-II) demonstrated that in presence of measurement errors, reciprocal of measured parallaxes $(1 / p)$ is not a good estimator for distances $r$. Instead, Bayesian approach with proper choice of prior need to be used to infer distance $r$ from the measured parallaxes $p$ together with it uncertainty $\sigma_{p}$. ABJ-II recommended two priors: a exponential decreasing space density prior and a prior based on Milky Way model. Later, these priors were applied to DR1 parallaxes by Astraatmadja \& Bailer-Jones (2016b, hereafter ABJ-III), who derived distances $r$ to the stars in TGAS. 

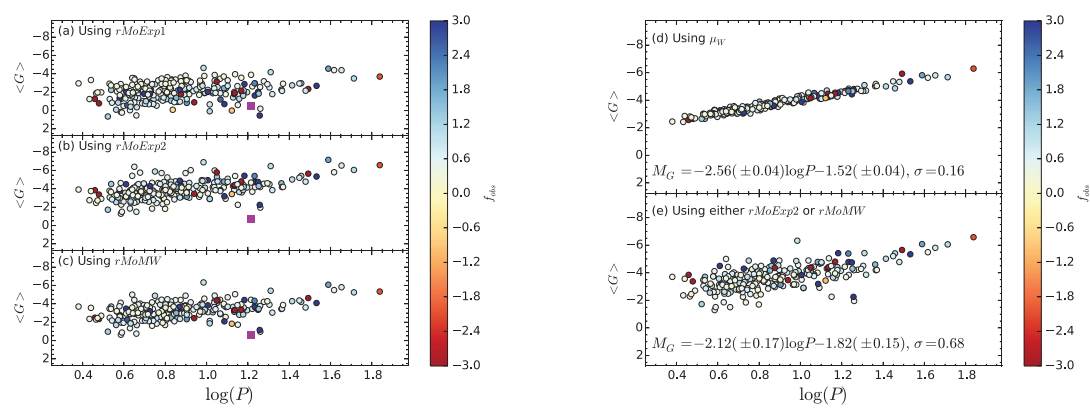

Figure 1. The $G$-band PL relation for Galactic Cepheids with difference adopted distances. The magenta square in the left panel is for RW Cam, the outlier in the comparison of parallaxes as shown in Casertano et al. (2016). Color bar represents the value of $f_{o b s}=\sigma_{p} / p$, restricted to $[-3,3]$ for display purpose. There are some Cepheids with $f_{o b s}>|3|$, shown as darkest blue/red colors. The average $f_{o b s}$ for this sample is 0.22 . Error bars are omitted for clarity.

We cross-matched catalog in ABJ-III to 400 Galactic Cepheids listed in Ngeow (2012, hereafter N2012), and 246 Cepheids were found matched in both catalogs.

The absolute magnitudes in Gaia G-band for the matched Galactic Cepheids were derived using four available distances or distance modulus: (1) distance modulus based on a calibrated period-Wesenheit relation given in N2012, $\mu_{W} ;(2)$ inferred distance based on the exponentially decreasing space density prior with a scale height $L=0.11 \mathrm{kpc}$, rMoExp1; (3) same as (2) but with $L=1.35 \mathrm{kpc}, r M o E x p 2$; and (4) inferred distance based on the Milky Way prior, $r M o M W$. The mode distances were adopted for (2)-(4). The $G$-band extinctions were estimated via $A_{G} / A_{V}=0.695$ (ABJ-II), where $A_{V}=3.23 E(B-V)$ and $E(B-V)$ is taken from N2012. Fig. 1(a)-(c) displays the resulted $G$-band PL relation, which shows that the PL relation based on the inferred distances from DR1 parallaxes, given in ABJ-III catalog, displays large scatter when compared to the PL relation constructed using the $\mu_{W}$ distance modulus from N2012 (Fig. 1[d]) . These PL relations are also shallower, and in general fainter (especially the one based on the $r M o E x p 1$ distance), than the PL relation shown in the Fig. 1(d). ABJ-III found that when $r<2000 \mathrm{pc}, r M o M W$ give a better agreement to distances based on external method; while for $r>2000 \mathrm{pc}, r M o E x p 2$ shows a better result. Assume $\mu_{W}$ gives the true distance, we adopted either $r M o M W$ or $r M o E x p 2$ based on the true distance. The revised PL relation is shown in Fig. 1(e) with reduced scatter. In conclusion, distances inferred from DR1 parallaxes are not suitable to calibrate the PL relation for Galactic Cepheids, and future Data Releases from Gaia are needed to improve such calibration.

This work has made use of data from the European Space Agency (ESA) mission Gaia (https://www.cosmos.esa.int/gaia), processed by the Gaia Data Processing and Analysis Consortium (DPAC, https://www.cosmos.esa.int/web/gaia/dpac/ consortium). Funding for the DPAC has been provided by national institutions, in particular the institutions participating in the Gaia Multilateral Agreement.

\section{References}

Astraatmadja, T. L. \& Bailer-Jones, C. A. L. 2016a, The Astrophysical Journal, 832, 137

Astraatmadja, T. L. \& Bailer-Jones, C. A. L. 2016, The Astrophysical Journal, 833, 119

Casertano, S., Riess, A. G., Bucciarelli, B., \& Lattanzi, M. G. 2016, Astronomy \& Astrophysics, 599,67

Lindegren, L., Lammers, U., Bastian, U., et al. 2016, Astronomy $\&$ Astrophysics, 595, A4

Ngeow, C.-C. 2012, The Astrophysical Journal, 747, 50 